\title{
Unit cell dependence of optical matrix elements in tight-binding theory: The case of zigzag graphene nanoribbons
}

\author{
Kondayya Gundra, ${ }^{1}$ and Alok Shukla ${ }^{1}$ \\ ${ }^{1}$ Department of Physics, Indian Institute of Technology, Bombay, Mumbai 400076 INDIA
}

\begin{abstract}
In the tight-binding theory, momentum matrix elements (MMEs) needed to calculate the optical properties are normally computed using a formulation based on the gradient of the Hamiltonian in the $\mathbf{k}$ space. We demonstrate the inadequacy of this formulation by considering the case of zigzag graphene nanoribbons. We show that one obtains wrong values of MMEs, in violation of the wellknown selection rules, if the unit cell chosen in the calculations does not incorporate the symmetries of the bulk. This is in spite of the fact that the band structure is insensitive to the choice of the unit cell. We substantiate our results based on group-theoretic arguments. Our observations will open an avenue for proper formulation of MMEs.
\end{abstract}

Keywords: Tight binding theory, Momentum matrix elements, Optical properties, Graphene nanoribbons

\section{INTRODUCTION}

Tight-binding theory is one of the conceptually simplest, and widely used theories of the electronic structure of molecules, clusters, and solids. And indeed, the newly emerging field of graphene[1] and related materials [2 6] such as graphene nanoribbons (GNRs) [7-10] has seen widespread use of the tight-binding approach for calculation of their electronic structure, [11 [15] and optical properties. [16] However, because of the unknown nature of the basis set associated with the tight-binding approach, the calculation of MMEs needed for computing the optical properties has always been a matter of debate. Blount [17] argued that the momentum operator for a periodic system can be represented as

$$
\mathbf{p}=\frac{m_{0}}{\hbar} \nabla_{\mathbf{k}} H(\mathbf{k}),
$$

where $m_{0}$ is the free electron mass, and $\nabla_{\mathbf{k}} H(\mathbf{k})$, represents the gradient of the Hamiltonian in the $\mathbf{k}$ space. This expression was used by Dresselhaus and Dresselhaus[18] as well as Smith[19] to perform early calculations of the optical properties of solids using the tight-binding approach. Based upon generalized Hellmann-Feynman theorem, Lew Yan Voon and Ram-Mohan, 20] argued that Eq. [1] is indeed the correct representation of the momentum operator for calculating optical properties. Adopting a gauge-invariant approach within the tight-binding formalism, Graf and Vogel[21] obtained results in agreement with the work of Lew Yan Voon and Ram-Mohan, 20]. Cruz et al. 222] also indicated that, Eq. 1 leads to the correct computation of optical matrix elements. However, in a recent analysis, Pedersen et al. 23] pointed out that Eq. [1 is incomplete, in that it does not contain the contribution of intra-atomic matrix elements. According to Pedersen et al.,23] the MMEs $\langle c(\mathbf{k})|\mathbf{p}| v(\mathbf{k})\rangle$ between the valence band states $(|v(\mathbf{k})\rangle)$ and the conduction band states $(|c(\mathbf{k})\rangle)$ is given by

$$
\begin{aligned}
\langle c(\mathbf{k})|\mathbf{p}| v(\mathbf{k})\rangle= & \frac{m_{0}}{\hbar} \sum_{\alpha \beta} C_{c \beta}^{*}(\mathbf{k}) C_{v \alpha}(\mathbf{k}) \nabla_{\mathbf{k}}\langle\beta, \mathbf{k}|H| \alpha, \mathbf{k}\rangle \\
& +\frac{i m_{0}\left(E_{c, \mathbf{k}}-E_{v, \mathbf{k}}\right)}{\hbar} \sum_{\alpha \beta} C_{c \beta}^{*}(\mathbf{k}) C_{v \alpha}(\mathbf{k}) \mathbf{d}_{\beta \alpha}
\end{aligned}
$$

where, the valence band eigen state $|v(\mathbf{k})\rangle$ is expressed as

$$
|v(\mathbf{k})\rangle=\sum_{\alpha} C_{v \alpha}(\mathbf{k})|\alpha, \mathbf{k}\rangle ;|\alpha, \mathbf{k}\rangle=\frac{1}{\sqrt{N}} \sum_{\mathbf{R}} e^{i \mathbf{k} . \mathbf{R}}|\alpha, \mathbf{R}\rangle
$$

*Permanent Address: Theoretical Physics Division, Bhabha Atomic Research Centre, Mumbai 400085, INDIA; Electronic address: naiduk@barc.gov.in, shukla@iitb.ac.in 
and a similar expression holds for the conduction band states $|c(\mathbf{k})\rangle$. In the equations above, $\mathbf{R}$ denotes a lattice vector, $N$ is the total number of unit cells in the system, $|\alpha, \mathbf{R}\rangle$ is the $\alpha$-th atomic orbital located in the unit cell at position $\mathbf{R}, E_{c, \mathbf{k}}\left(E_{v, \mathbf{k}}\right)$ is the energy eigen value of the conduction (valence) band and $\mathbf{d}_{\beta \alpha}=\langle\beta, 0|\mathbf{r}| \alpha, 0\rangle$ is the matrix element of the position operator $\mathbf{r}$ with respect to the reference unit cell. Note that the second term on the right hand side (r.h.s.) of Eq. 2, called intra-atomic contribution[23], is the extra term as compared to Eq. 1. Sandu[24] further examined the issue and essentially agreed with the analysis of Pedersen et al. [23]

Recently, while developing a correlated electron approach for computing optical properties of GNRs [25], we discovered that the optical matrix elements of zigzag GNRs (ZGNRs) computed using Eqs. 10 or 2 were crucially depend on the choice of the unit cell. While the band structures of the ZGNRs in question, as expected, were found to be independent of the nature of the unit cell, however, correct values of optical matrix elements were not obtained unless the chosen unit cell also incorporated the point-group symmetry of the bulk. This result is counter-intuitive, and surprising, because normally we believe that the computed physical quantities for bulk systems should be independent of the choice of the unit cell. This issue is particularly important for the case of ZGNRs for which there is a certain ambiguity in the choice of unit cells, as compared to the case of armchair GNRs (AGNRs) for which a unique choice of the unit cell exists. To the best of our knowledge, this unit cell dependence of the formalism based on Eqs. 1 or 2. has not been reported earlier, therefore, here we aim to elaborate our findings, and to analyze our results, based upon group theoretic arguments.

Remainder of this paper is organized as follows. In the next section we discuss the theoretical aspects of this work. In particular, we analyse the nature of unit cells chosen for the calculations from the point of view of their point group symmetries, and, based on their irreducible representations, deduce the optical selection rules. In section III we present and analyse our results. In particular, we find that the results obtained are fully consistent with the optical selection rules deduced in section [I] Furthermore, we also support our arguments by means of finite cluster calculations. Finally, in section IV we present our conclusions.

\section{THEORY}

In the present work, we consider a nearest-neighbor tight-binding (TB) model for GNRs, with zero site energies

$$
H=\sum_{\langle i j\rangle, \sigma} t_{i, j}\left(c_{i \sigma}^{\dagger} c_{j \sigma}+c_{j \sigma}^{\dagger} c_{i \sigma}\right),
$$

where $\langle i j\rangle$ implies nearest neighbors $(\mathrm{NN}), c_{i \sigma}^{\dagger}$ creates an electron of spin $\sigma$ on the $p_{z}$ orbital of carbon atom $i$ (assuming that the ribbon lies in the $x y$-plane, with the $x$-axis being the periodicity direction), and $t_{i j}$ is the corresponding hopping matrix element. In order to obtain the band structure and the corresponding Bloch orbitals, the TB Hamiltonian of Eq. 4 is Fourier transformed, and the corresponding matrix elements for the one dimensional (1D) system under consideration are obtained as

$$
H_{i, j}(k)=\sum_{n=-\infty}^{n=+\infty} e^{i k n a} t_{i(n a), j(0)}
$$

where $i(n a)$ represents the $i$-th orbital of the unit cell located at position $n a, n$ being an integer and $a$ is the lattice constant, $j(0)$ represents the $j$-th orbital of the reference unit cell, and $t_{i(n a), j(0)}$ is the corresponding hopping element which is non-zero only for the NN sites. The Hamiltonian obtained from Eq. 5 is diagonalized at different $k$-points to obtain the band structure and the corresponding Bloch orbitals in the 1D Brillouin zone. In order to compute the optical absorption spectrum within the TB model, the MMEs $\langle c(\mathbf{k})|\mathbf{p}| v(\mathbf{k})\rangle$ need to be computed. For the purpose, we have used the formula proposed by Pedersen et al. [23] (cf. Eq. 2), as against the original approach of Blount [17] embodied in Eq. 1. In order to ensure the correctness of the approaches, we performed calculations of the matrix element $\left\langle c(\mathbf{k})\left|p_{x}\right| v(\mathbf{k})\right\rangle$ needed to compute the absorption spectrum for the light polarized along the $x$-direction, using both Eqs. 1, and 2, and found only quantitative differences. However, Eq. 22 is more general, and can also be used to compute the matrix element $\left\langle c(\mathbf{k})\left|p_{y}\right| v(\mathbf{k})\right\rangle$ required for calculating the absorption spectrum for the $y$-polarized light, by setting the first term on its r.h.s. to zero, because for a 1D system periodic along the $x$ direction, the Hamiltonian has no $k_{y}$ dependence.

For the present 1D systems, we used the optical matrix elements to compute the optical absorption spectrum for the $x$-polarized ( $y$-polarized) photons in form of the corresponding components of the imaginary part of the dielectric constant tensor, i.e., $\epsilon_{x x}^{(2)}\left(\epsilon_{y y}^{(2)}(\omega)\right)$, using the standard formula

$$
\epsilon_{i i}^{(2)}(\omega)=C \sum_{v, c} \int_{-\pi / a}^{\pi / a} \frac{\left|\left\langle c(k)\left|p_{i}\right| v(k)\right\rangle\right|^{2}}{\left\{\left(E_{c v}(k)-\hbar \omega\right)^{2}+\gamma^{2}\right\} E_{c v}^{2}(k)} d k,
$$




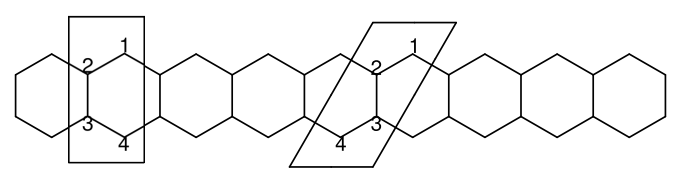

(a)

(b)

Figure 1: ZGNR-2, along with the two possible choices of unit cells which can generate it. The $x$-axis is taken along the periodicity direction, $y$-axis is perpendicular to it within the plane of the system, while the $z$-axis is perpendicular to that plane. Symmetry groups of cells (a) and (b) are $C_{2 v}$ and $C_{i}$, respectively. Based upon the higher symmetry group of cell (a) we call it the symmetric cell, and (b) the asymmetric cell. The numbering of the atoms is consistent with the Hamiltonian matrices given in Eqs. 7 and 8 .

where $i$ denotes the Cartesian direction in question, $\omega$ represents the angular frequency of the incident radiation, $E_{c v}(k)=E_{c, k}-E_{v, k}, \gamma$ is the line width, while $C$ includes rest of the constants. We have set $C=1$ in all the cases to obtain the absorption spectra in arbitrary units.

We will consider the optical matrix elements of various ZGNRs, characterized by their width parameter $N_{Z}$, which is nothing but the number of zigzag lines across the width of the ribbon. In short, we will denote a ZGNR of width $N_{Z}$ as ZGNR- $N_{Z}$. We first consider the narrowest such ribbon ZGNR-2, shown in Fig. 1, which has four carbon atoms per unit cell and can be generated by periodically repeating either of the two different unit cells depicted in the same figure. Even though both types of unit cells lead to the same GNR in the bulk limit, the point group symmetries of the two unit cells are different. The unit cell of Fig. 1a has $C_{2 v}$ symmetry with the symmetry operators (in addition to the identity): (i) rotation by $180^{\circ}$ about the $x$-axis, (ii) reflection about the $x y$ plane, and (iii) reflection about the $x z$ plane, leading to four irreducible representations (irreps) $A_{1}, A_{2}, B_{1}$, and $B_{2}$.[27] As per dipole selection rules of the $C_{2 v}$ point group, $x$-polarized radiation will couple bands with $A_{1}$ symmetry while the $y$-polarized radiation will couple $A_{1}$ states to $B_{1}$ states. 27] On the other hand, the unit cell of Fig. 10, has $C_{i}$ symmetry with only the identity and the inversion being the symmetry operators. This group has irreps $A_{g}$ and $A_{u}$, and dipole selection rules allow $x, y$, and $z$, polarized (and mixtures thereof) radiation to cause optical transitions between $A_{g}$ and $A_{u}$ bands. Thus, the symmetry analysis predicts that for the $C_{2 v}$ unit-cell (Fig. 1 1 ), the optical matrix elements will have either $x$ - or $y$ - non-zero components, while for the $C_{i}$ case (Fig. 1b) both the components will be simultaneously nonzero.

Using Eq. 5, and assuming that the NN hopping matrix element is $t$, the Hamiltonian matrix for the symmetric unit cell (cf. Fig. 19) is obtained to be

$$
H_{S}(k)=\left(\begin{array}{cccc}
0 & t\left(1+e^{-i k a}\right) & 0 & 0 \\
t\left(1+e^{i k a}\right) & 0 & t & 0 \\
0 & t & 0 & t\left(1+e^{i k a}\right) \\
0 & 0 & t\left(1+e^{-i k a}\right) & 0
\end{array}\right),
$$

while for the asymmetric unit cell (Fig. 10) the corresponding matrix is

$$
H_{A S}(k)=\left(\begin{array}{cccc}
0 & t\left(1+e^{-i k a}\right) & 0 & 0 \\
t\left(1+e^{i k a}\right) & 0 & t & 0 \\
0 & t & 0 & t\left(1+e^{-i k a}\right) \\
0 & 0 & t\left(1+e^{i k a}\right) & 0
\end{array}\right) .
$$

In order to compute the optical matrix elements (cf. Eq. 2), we need the matrix elements of the $\nabla_{\mathbf{k}} H(\mathbf{k})$ operator, which for the present 1D case, can be obtained easily by taking the derivative of each element of $H_{S}(k) / H_{A S}(k)$, with respect to $k$. Furthermore, the $\mathbf{d}$ matrix ( $c f$. Eq. 2) needed for the purpose is taken to be diagonal, with its elements being the Cartesian coordinates of various atoms of the reference unit cell.

Next, we demonstrate that that the Hamiltonian matrices of Eqs. 7 and 8 are unitarily equivalent. Let us consider the $k$-dependent unitary transformation

$$
U(k)=\left(\begin{array}{cccc}
1 & 0 & 0 & 0 \\
0 & 1 & 0 & 0 \\
0 & 0 & 1 & 0 \\
0 & 0 & 0 & e^{i k a}
\end{array}\right) .
$$

It is straightforward to show that $H_{S}(k)=U(k)^{\dagger} H_{A S}(k) U(k)$. Therefore, it is obvious that both $H_{S}(k)$ and $H_{A S}(k)$, will yield the identical band structure. 
Figure 2: Band structure of ZGNR-2 (cf. Fig. 1) obtained by diagonalizing either $H_{S}(k)$ or $H_{A S}(k)(c f$. Eqs. 7 and 8$)$ with $t=-2.6 \mathrm{eV}$. Valence bands are labeled as $\mathrm{v}_{1} / \mathrm{v}_{2}$ and conduction bands as $\mathrm{c}_{1} / \mathrm{c}_{2}$.

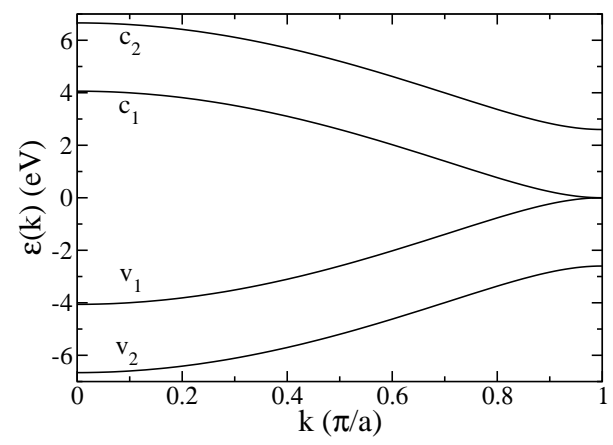

Table I: Irreps of the Bloch orbitals of ZGNR-2 at $k=0$ and $k=\pi / a$, for the symmetric and the asymmetric choices of the unit cell. The Bloch orbitals are presented in the appendix.

\begin{tabular}{|c||c|c|c|c|}
\hline \multicolumn{1}{|c||}{ Band } & \multicolumn{3}{c|}{ Irreps of the Bloch orbitals } \\
\hline \multirow{2}{*}{} & \multicolumn{2}{c|}{$k=0$} & \multicolumn{2}{c|}{$k=\pi / a$} \\
\cline { 2 - 5 } & Sym $\left(C_{2 v}\right)$ & Asym $\left(C_{i}\right)$ & $\operatorname{Sym}\left(C_{2 v}\right)$ & Asym $\left(C_{i}\right)$ \\
\hline \hline$v_{2}$ & $A_{1}$ & $A_{g}$ & $A_{1}$ & $A_{g}$ \\
\hline$v_{1}$ & $B_{1}$ & $A_{u}$ & $B_{1}$ & $A_{g}$ \\
\hline$c_{1}$ & $A_{1}$ & $A_{g}$ & $A_{1}$ & $A_{u}$ \\
\hline$c_{2}$ & $B_{1}$ & $A_{u}$ & $B_{1}$ & $A_{u}$ \\
\hline
\end{tabular}

\section{RESULTS AND DISCUSSION}

Here we present and discuss our numerical results in two subsections, organized as follows: the first subsection contains the results for various ribbons in the bulk limit (infinite length), while in the second one the results are presented for finite-clusters of increasing sizes for the two choices of the unit cell. As shown below, the finite-cluster calculations help us in obtaining a better understanding of the results for the infinitely long ribbons.

\section{A. Infinite Ribbons}

The band structure of ZGNR-2 obtained by diagonalizing either $H_{S}(k)$ or $H_{A S}(k)$ is identical, and is presented in Fig. 2 for $t=-2.6 \mathrm{eV}$. The irreps of the corresponding Bloch orbitals at $k=0$ and $k=\pi / a$, for the two choices of the unit cell are listed in Table 1 , and can be easily deduced from the Bloch orbitals given in the appendix.

In Table [I] we present the values of optical matrix elements between different bands for both the symmetric and asymmetric unit cell cases, and for both types of unit cells the calculations were performed with Eq. 2, It is a wellknown fact that in ZGNRs with even values of $N_{Z}$, and for light polarized along the periodicity direction ( $x$-axis), HOMO-LUMO transition is forbidden, as are those between several other bands due to symmetry related selection rules. [16] Combining the knowledge of the irreps of various Bloch orbitals ( $c f$. Table【) at different $k$-points, with the dipole selection rules of point groups $C_{2 v}$ discussed in section II we note from table II that, consistent with these selection rules, the $x$-component of the optical matrix elements between bands $c_{1}-v_{1}$ (HOMO-LUMO) vanishes only if the symmetric unit cell is considered for the system. From the same table it is also obvious this transition is allowed for the $y$-polarized radiation when the symmetric cell is used, consistent again with the selection rules of the $C_{2 v}$ point group. While, with the asymmetric unit cell, both the $x$ and $y$ components of the optical matrix element are found to be non-zero for the transition, a result in agreement with the $C_{i}$ symmetry of the asymmetric cell. Thus, we obtain different results for the polarization characteristics of the radiation for the $c_{1}-v_{1}$ transition: (a) with the symmetric cell it is strictly $y$ polarized, while (b) with the asymmetric cell it has both $x$ and $y$ components. As far as the $c_{2}-v_{1}$ (and $c_{1}-v_{2}$ ) transitions are concerned both the unit cells predict it to be $x$ polarized, however, the magnitude of the optical matrix element obtained with the asymmetric unit cell is much smaller as compared to that with the symmetric cell. 
Figure 3: Components of the imaginary part of the frequency dependent dielectric constant tensor of ZGNR-2, calculated using $t=-2.6 \mathrm{eV}:(\mathrm{a}) \epsilon_{x x}^{(2)}(\omega)$ for the symmetric unit cell (solid line), and asymmetric unit cell (broken line), and (b) $\epsilon_{y y}^{(2)}(\omega)$ is identical for both the unit cells. A line width of $0.05 \mathrm{eV}$ was used to plot the spectrum.
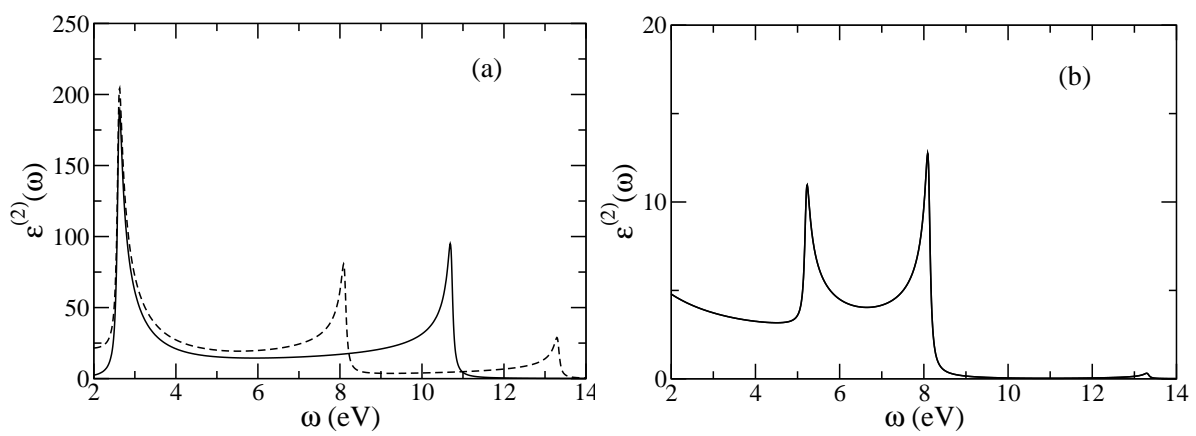

In Fig. 3 we present the optical absorption spectrum of ZGNR-2, for both the $x$ - and $y$-polarized radiation, calculated for the two choices of the unit cell. From the band structure of ZGNR-2 (cf. Fig. 2) it is obvious that the joint density of states (JDOS) of the conduction and valence bands have van Hove ( $\mathrm{vH})$ singularities at points $k=0$ and $k=\pi / a$, because of the parallel bands. This implies that, the optical absorption spectrum for the $x$-polarized light, will have peaks corresponding to gaps between those bands at points $k=0$ and $k=\pi / a$, for which the transition is allowed by the selection rules. Therefore, we expect two peaks in $\epsilon_{x x}^{(2)}(\omega)$ for ZGNR-2: (a) first peak around $2.5 \mathrm{eV}$ corresponding to $c_{1}-v_{2} / c_{2}-v_{1}$ allowed transitions at $k=\pi / a$, and (b) the second peak close to $11 \mathrm{eV}$ because of the transition between the same bands at $k=0$. For the $y$-polarized radiation, based upon the selection rules and the $\mathrm{vH}$ singularities we expect three peaks in $\epsilon_{y y}^{(2)}(\omega)$ : (a) first one near $5 \mathrm{eV}$ due to the $c_{2}-v_{2}$ transition at $k=\pi / a$, (c) second one close to $8 \mathrm{eV}$ due to the $c_{1}-v_{1}$ transition at $k=0$, and (d) the final one near $13 \mathrm{eV}$ caused by the $c_{2}-v_{2}$ transition also at $k=0$.

An inspection of Fig 3 b reveals that the calculated values of $\epsilon_{y y}^{(2)}(\omega)$ exhibit precisely the three peaks (the last peak near $13 \mathrm{eV}$ being barely visible) described above, both, for the symmetric, as well as for the asymmetric, unit cells. The fact that the results for $\epsilon_{y y}^{(2)}(\omega)$ are same with both choices of the unit cell is fairly obvious because the optical matrix elements needed to compute $\epsilon_{y y}^{(2)}(\omega)$ depend only on the $y$ coordinates of the sites (cf. Eq. 2), which are identical for both the unit cells. This is also obvious from the $y$-components of the optical matrix elements listed for various $k$-points in Table II.

For $\epsilon_{x x}^{(2)}(\omega)$, however, the situation is different. The calculated spectrum for the symmetric unit cell is fully consistent with the $\mathrm{vH}$ singularity based analysis of the JDOS, with the two peaks precisely at the predicted locations in Fig. 3a. However, in the same figure, the spectrum computed with the asymmetric unit cell agrees with this picture only for the first peak, while it contains two higher energy peaks located near $8 \mathrm{eV}$ and $13 \mathrm{eV}$, in complete disagreement with the correct spectrum. From Table I it is obvious that the optical matrix elements for both the symmetric and asymmetric unit cells are identical at the point $k=\pi / a$, therefore, even with the asymmetric unit cell we get the correct description of the first peak in $\epsilon_{x x}^{(2)}(\omega)$. However, the peaks around $8 \mathrm{eV}$ and $13 \mathrm{eV}$ in the asymmetric cell spectrum correspond to $c_{1}-v_{1}$ and $c_{2}-v_{2}$ transitions, respectively, at $k=0$, which have become allowed because of the incorrectly calculated values of the corresponding optical matrix elements ( $c f$. Table II $)$. Thus, the peak around $8 \mathrm{eV}$ is now present both in $\epsilon_{x x}^{(2)}(\omega)$ and $\epsilon_{y y}^{(2)}(\omega)$ computed with the asymmetric cell, leading to absorption with mixed polarization, a wrong result as discussed earlier. Furthermore, the peak close to $11 \mathrm{eV}$ is missing from the asymmetric cell spectrum because for that case transitions $c_{1}-v_{2} / c_{2}-v_{1}$ have become wrongly disallowed at $k=0$ ( $c f$. Table II). Thus we conclude that with the asymmetric unit cell, for ZGNR-2 while one obtains the correct description of the lowest peak in the absorption spectrum, the predictions for the higher energy peaks in the spectrum are completely wrong.

In order to ensure that the results presented here for the ZGNR-2 are universal, and not just valid for this particular ZGNR, we also performed similar calculations for the ZGNR-12. The symmetric and asymmetric unit cells considered for this ZGNR are presented in Fig. 4, and again the symmetric cell has $C_{2 v}$ symmetry while the asymmetric one possesses $C_{i}$ symmetry. For both types of unit cells we obtain the identical band structure (not presented here), which is in agreement with that presented by other authors.[16] Because the number of bands, and, therefore, the number of possible optical matrix elements is quite large for this case, in Fig. 5 we directly present the optical absorption 
Table II: Optical matrix elements of ZGNR-2 between the bands identified in Fig. 2 at various points in the Brillouin zone, with $k$ expressed in the units of $\pi / a$. Column heading "Sym" corresponds to the values for the symmetric unit cell (Fig. 1 7 ) and "Asym" denotes the results obtained for the asymmetric unit cell (Fig. 10).

\begin{tabular}{|c|c|c|c|c|c|c|c|c|}
\hline$k(\pi / a)$ & $\left\langle c_{1}\left|p_{x}\right| v_{1}\right\rangle$ & $\left\langle c_{1}\left|p_{y}\right| v_{1}\right\rangle$ & \multicolumn{2}{|c|}{$\left\langle c_{2}\left|p_{x}\right| v_{1}\right\rangle$} & \multicolumn{2}{|c|}{$\left\langle c_{2}\left|p_{y}\right| v_{1}\right\rangle$} \\
\hline \hline & Sym & Asym & Sym & Asym & Sym & Asym & Sym & Asym \\
\hline 0.0 & 0.00 & 25.45 & 4.10 & 4.10 & 27.04 & 0.00 & 0.00 & 0.00 \\
\hline 0.2 & 0.00 & 22.88 & 3.77 & 3.77 & 24.50 & 0.04 & 0.00 & 0.00 \\
\hline 0.5 & 0.00 & 12.02 & 2.25 & 2.25 & 13.90 & 0.38 & 0.00 & 0.00 \\
\hline 0.8 & 0.00 & 1.56 & 0.41 & 0.41 & 5.00 & 2.42 & 0.00 & 0.00 \\
\hline 1.0 & 0.00 & 0.00 & 0.00 & 0.00 & 6.76 & 6.76 & 0.00 & 0.00 \\
\hline
\end{tabular}

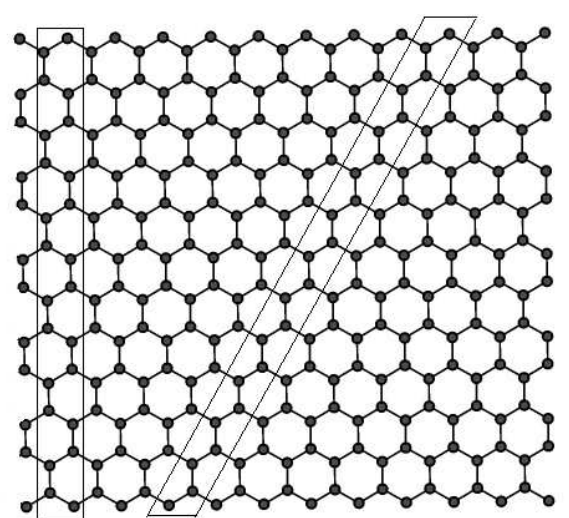

(a) (b)

Figure 4: Unit cells considered for the ZGNR-12: (a) symmetric unit cell, and (b) asymmetric unit cell.

spectra for the $x$-polarized photons $\left(\epsilon_{x x}^{(2)}(\omega)\right)$ of this ribbon computed using both types of unit cells.

From the figure, a trend similar to the case of ZGNR-2 is observed as far as the agreement between the spectra obtained using symmetric and asymmetric unit cells are concerned. The two sets of spectra agree at lower energy peaks, but diverge completely from each in the higher energy region. As compared to the symmetric cell spectrum, the one computed with the asymmetric cell in the high energy region has many missing peaks as well as several new peaks. The reasons behind this are precisely the same as for the case of ZGNR-2, that optical matrix elements are being wrongly computed with the asymmetric cell, thus allowing disallowed transitions and vice verse, leading to a spectrum in complete disagreement with the published results. [16] In order to ascertain whether this behavior was an artifact of the tight-binding model, we included electron-electron interactions by employing the Pariser-Parr-People (PPP) model Hamiltonian[28] to perform similar comparisons. Although the results of our PPP model based work will be discussed in detail elsewhere, [25]but we again found this unit-cell anomaly while computing the optical matrix

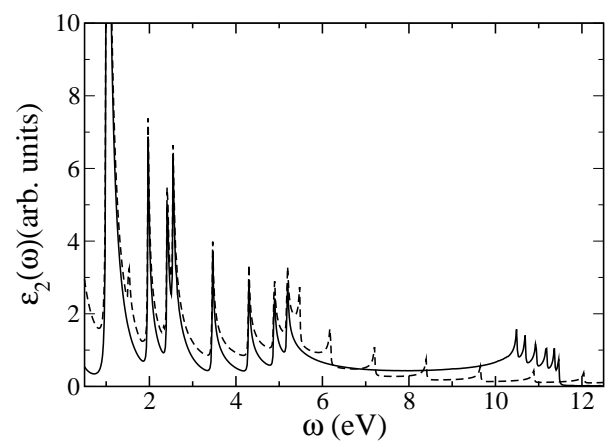

Figure 5: $\epsilon_{x x}^{(2)}(\omega)$ of ZGNR-12, computed using $t=-2.6 \mathrm{eV}$, for the symmetric (solid line) and the asymmetric (broken line) unit cells. A line width of $0.01 \mathrm{eV}$ was used to plot the spectrum. 
Table III: HOMO-LUMO gap $E_{g}$ (in eV), $x$ - and $y$ - components of dipole transition matrix elements $\langle L|x| H\rangle$, and $\langle L|y| H\rangle$ between the HOMO and LUMO orbitals, respectively (in $\AA$ units), for finite clusters containing $N$ primitive cells of the symmetric and asymmetric types.

\begin{tabular}{|c|c|c|c|c|c|c|}
\hline$N$ & \multicolumn{3}{|c|}{ Symmetric Cell } & \multicolumn{3}{c|}{ Asymmetric Cell } \\
\hline & $E_{g}$ & $\langle L|x| H\rangle / N$ & $\langle L|y| H\rangle$ & $E_{g}$ & $\langle L|x| H\rangle / N$ & $\langle L|y| H\rangle$ \\
\hline 25 & 0.0197 & 0.000 & 1.392 & 0.0269 & 0.769 & 1.386 \\
\hline 50 & 0.0050 & 0.000 & 1.398 & 0.0070 & 0.757 & 1.396 \\
\hline 100 & 0.0013 & 0.000 & 1.400 & 0.0018 & 0.751 & 1.399 \\
\hline 200 & 0.0003 & 0.000 & 1.399 & 0.0005 & 0.747 & 1.400 \\
\hline
\end{tabular}

Figure 6: Finite clusters containing $N$ repeat units of: (a) Symmetric cell, and (b) Asymmetric cell

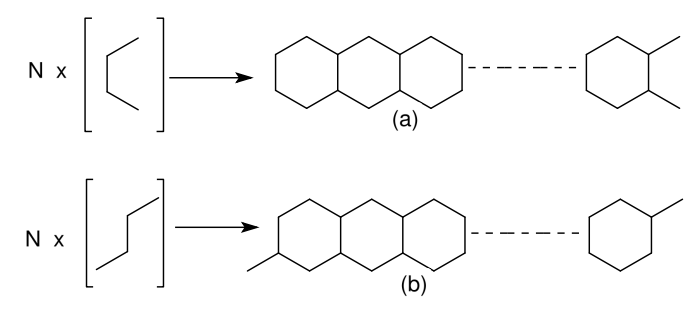

elements.

\section{B. Finite-Cluster Calculations}

ZGNR-2, with hydrogenated edges is nothing but the polymer polyacene, which has so far not been synthesized. [26] However, several oligomers of polyacene (oligoacenes) such as naphthalene, anthracene, tetracene, pentacene, and hexacene are known to exist, and their properties have been studied extensively both theoretically and experimentally. 26] The symmetry group of oligoacenes is $D_{2 h}$, and the optical transitions in it are either $x$ - or $y$-polarized. 26] The HOMOLUMO transition in oligoacenes is always $y$-polarized, just as in ZGNR-2, while the dominant $x$-polarized transition is among the orbitals HOMO(LUMO) and LUMO $+n(\mathrm{HOMO}-n)$, where $n(\geq 1)$ is an integer which depends on the oligoacene in question. 26] Noting that, the edge hydrogenation does not change the point group of the system, optical selection rules of ZGNR-2 and polyacene will be identical. Just as an oligoacene with an infinite number of repeat units is polyacene, an infinite number of repeat units of either the symmetric cell or the asymmetric cell will lead to ZGNR-2. Therefore, in what follows, we investigate the optical properties of clusters of increasing sizes, consisting of finite number of unit cells of the two types, to understand their evolution towards the bulk ZGNR-2. The shape of the finite clusters for the two cases is shown in Fig. 6. From the figure it is obvious that, because of the dangling bonds at the edges, the point groups of the clusters are $C_{2 v}$ and $C_{i}$, for the symmetric and the asymmetric cases, respectively. Furthermore, without the dangling bonds, the symmetry group of these clusters will be $D_{2 h}$, just as in the case of oligoacenes.

Table III contains our results on the HOMO-LUMO gap, $E_{g}$, HOMO-LUMO dipole transition matrix elements $\langle L|x| H\rangle / N[29]$, and $\langle L|y| H\rangle$ for the increasing cluster sizes. The transition dipole moments were computed using the methodology adopted in our earlier work. [26] From the table it is obvious that: (a) For both types of clusters, the gap $E_{g}$ is slowly closing with increasing $N$, (b) $\langle L|y| H\rangle$ is virtually the same for both types of clusters, and (c) $\langle L|x| H\rangle / N$ is exactly zero for all $N$ for the symmetric case, but it has significant non-zero values for the asymmetric case, and demonstrates a slow decrease with increasing $N$. This confirms the results of the previous section that both types of unit cells yield the same band structure in the bulk limit, but different values of the $x$ components of the transition dipole moments.

\section{CONCLUSIONS}

In this work, we investigated the influence of the nature of the primitive cells of ZGNRs on their electronic structure and optical properties, as computed within the tight-binding model. Based upon the Bloch orbital based calculations for the bulk systems, as well on finite clusters of increasing sizes, we conclude that the choice of the unit cell is 
of crucial importance when it comes to evaluation of optical matrix elements within $k$-space gradient formulation, although, it is inconsequential as far as the band structure is concerned. In other words, Hamiltonians which are unitarily equivalent, lead to different values of optical transition matrix elements. This result is surprising because the theory of the optical properties of a system should not depend on the choice of the unit cell adopted to describe it. Our work demonstrate the inadequacy of this routinely used formulation for evaluation of optical matrix elements. We further substantiated our findings based upon the group theoretical analysis of optical matrix elements, and conclude that a unit cell which does not have the symmetries of the bulk system, leads to erroneous values of optical matrix elements violating symmetry based selection rules. Therefore, in order to describe the optical properties correctly within the $k$-space gradient formulation, it is important to choose a unit-cell whose symmetry is consistent with that of bulk.

Acknowledgments: We thank the Department of Science and Technology (DST), Government of India, for providing financial support for this work under Grant No. SR/S2/CMP-13/2006. K. G is grateful to Dr. S. V. G. Menon (BARC) for his continued support of this work.

[1] K. S. Novoselov et al., Science 306, 666 (2004); K. S. Novoselov et al., Nature 438, 197 (2005).

[2] C. Berger, et al., J. Phys. Chem. B 108, 19912 (2004).

[3] Y. Zhang et al., Appl. Phys. Lett. 86, 073104 (2005).

[4] C. L. Kane et al., Phys. Rev. Lett. 95, 226801 (2005).

[5] N. M. R. Peres et al., Phy. Rev. B 73, 125411 (2006).

[6] Y. W. Son et al., Nature 444, 347 (2006).

[7] X. H. Zheng et al., Phys. Rev. B 80, 235426 (2009).

[8] J.-W. Rhim and K. Moon Phys. Rev. B 80, 155441 (2009).

[9] J. Jung and A. H. MacDonald, Phys. Rev. B 79, 235433 (2009).

[10] A. H. Castro Neto et al., Rev. Mod. Phys. 81, 109 (2009) and references there in.

[11] K. Nakada et al., Phys. Rev. B 54, 17954 (1996).

[12] M. Fujita et al., J. Phys. Soc. Jpn 65, 9120 (1996).

[13] M. Ezawa, Phys. Rev. B 73, 045432 (2006).

[14] A. Onipko, Phys. Rev. B 78, 245412 (2008).

[15] L. Malysheva and A. Onipko Phys. Rev. Lett. 100, 186806 (2008).

[16] H. Hsu and L. E. Reichl, Phys. Rev. B 76, 045418 (2007).

[17] E. I. Blount, Solid State Physics, edited by F. Seitz and D. Turnbull (Academic Press Inc., New York, 1962), Vol. 13, p. 305.

[18] G. Dresselhaus and M. S. Dresselhaus, Phys. Rev. 160, 649 (1967).

[19] N. V. Smith, Phys. Rev. B 19, 5019 (1979).

[20] L. C. Lew Yan Voon and L. R. Ram-Mohan, Phys. Rev. B 47, 15500 (1993).

[21] M. Graf and P. Vogl, Phys. Rev. B 51, 4940 (1995).

[22] M. Cruz, M. R. Beltrán, C. Wang, J. Tagüeña-Martínez, Y. G. Rubo, Phys. Rev. B 59, 15381 (1999).

[23] T. G. Pedersen, K. Pedersen, and T. B. Kriestensen, Phys. Rev. B 63, 201101(R) (2001).

[24] T. Sandu, Phys. Rev. B 72, 125105 (2005).

[25] K. Gundra and A. Shukla, Phys. Rev B 83, 075413 (2011).

[26] See, e.g., P. Sony and A. Shukla, Phys. Rev. B 75, 155208 (2007), and references therein for the works of other authors.

[27] See, e.g., M. Tinkham, Group theory and Quantum Mechanics (1964).

[28] R. Pariser and R. G. Parr, J. Chem. Phys. 21, 767 (1953); J. A. Pople, Trans Faraday Soc. 49, 1275 (1953).

[29] The $x$-component of the transition dipole was divided by $N$, because it increases almost linearly with the increasing cluster size. 
Table IV: Bloch orbital coefficients of ZGNR-2 at two $k$ points for the symmetric cell. For each orbital, basis functions are numbered 1 to 4, from top to bottom. For each coefficient, the real part is followed by its imaginary part.

\begin{tabular}{|c|c|c|c|c|c|c|c|}
\hline \multicolumn{4}{|c|}{$k=0$} & \multicolumn{4}{|c|}{$k=\pi / a$} \\
\hline$v_{2}$ & $v_{1}$ & $c_{1}$ & $c_{2}$ & $v_{2}$ & $v_{1}$ & $c_{1}$ & $c_{2}$ \\
\hline $0.44,0.00$ & $-0.56,0.00$ & $0.56,0.00$ & $-0.44,0.00$ & $0.00,0.00$ & $-0.71,0.00$ & $0.71,0.00$ & $0.00,0.00$ \\
\hline $0.56,0.00$ & $-0.44,0.00$ & $-0.44,0.00$ & $0.56,0.00$ & $0.00,0.71$ & $0.00,0.00$ & $0.00,0.00$ & $0.00,0.71$ \\
\hline $0.56,0.00$ & $0.44,0.00$ & $-0.44,0.00$ & $-0.56,0.00$ & $0.00,0.71$ & $0.00,0.00$ & $0.00,0.00$ & $0.00,-0.71$ \\
\hline $0.44,0.00$ & $0.56,0.00$ & $0.56,0.00$ & $0.44,0.00$ & $0.00,0.00$ & $0.71,0.00$ & $0.71,0.00$ & $0.00,0.00$ \\
\hline
\end{tabular}

Table V: Bloch orbital coefficients of ZGNR-2 at two $k$ points for the asymmetric cell. For each orbital, basis functions are numbered 1 to 4, from top to bottom. For each coefficient, the real part is followed by its imaginary part.

\begin{tabular}{|c|c|c|c|c|c|c|c|}
\hline \multicolumn{5}{|c|}{$k=0$} & \multicolumn{4}{c|}{$k=\pi / a$} \\
\hline$v_{2}$ & $v_{1}$ & $c_{1}$ & $c_{2}$ & $v_{2}$ & $v_{1}$ & $c_{1}$ & $c_{2}$ \\
\hline \hline $0.44,0.00$ & $-0.56,0.00$ & $0.56,0.00$ & $-0.44,0.00$ & $0.00,0.00$ & $0.71,0.00$ & $-0.71,0.00$ & $0.00,0.00$ \\
\hline $0.56,0.00$ & $-0.44,0.00$ & $-0.44,0.00$ & $0.56,0.00$ & $0.00,0.71$ & $0.00,0.00$ & $0.00,0.00$ & $0.00,0.71$ \\
\hline $0.56,0.00$ & $0.44,0.00$ & $-0.44,0.00$ & $-0.56,0.00$ & $0.00,0.71$ & $0.00,0.00$ & $0.00,0.00$ & $0.00,-0.71$ \\
\hline $0.44,0.00$ & $0.56,0.00$ & $0.56,0.00$ & $0.44,0.00$ & $0.00,0.00$ & $0.71,0.00$ & $0.71,0.00$ & $0.00,0.00$ \\
\hline
\end{tabular}

Appendix A: Bloch Orbitals of ZGNR-2 for the symmetric and asymmetric unit cells

The Bloch orbitals of ZGNR-2, obtained with the nearest neighbor hopping matrix element of $-2.6 \mathrm{eV}$ is presented in table IV for symmetric cell and in table $\mathrm{V}$ for asymmetric cell. 